\title{
Research on Communication Management based on Internet of Things
}

\author{
Junyan LI \\ Chinacomm Design \& Consulting Co., Ltd., Beijing, China
}

\begin{abstract}
The concepts and development prospects of Internet of Things is described briefly. The key technologies of hierarchical information are applied to the smart grid Internet of Things. The network congestion is prevented and the network performance is enhanced. Hence, the hierarchical information aggregation technology is one of the most important issues in the internet of things and smart grid.
\end{abstract}

KEYWORD: smart grid; the internet of things; information aggregation; data fusion; electricity market

\section{INTRODUCTION}

Information science and technology has produced three waves, is the emergence of the 1980 PCS for the first time, the second wave was the emergence of the Internet in 1995 and the third wave is the generation of the Internet of things. The Internet of things is the modern information technology development to a certain stage inevitable product, should is all roads lead to Rome and aggregation of modern technology, systematic innovation and revolution of information technology.

This paper describes the history and development of the Internet of things, definition, status and the architecture and key technology of Internet of things, and introduces the application of Internet of things. This article will guide you into the world of the Internet of things, feel the Internet of things run way, experience the world of the Internet of things.

\section{RESEARCH ON INTERNET OF THINGS IN SMART GRID}

\subsection{Internet of Things Principle}

The demands for great amount, clean, and green power energy rapidly grow for the global climate change. The Internet of Things is considered one of the very promising technologies for the implementation. As networking technology characteristics and development trend of gradually clear, the relationship between networking and smart grid is gradually clear.(1) Things is an important support for smart grid technologies. Things technology can improve the depth and breadth of comprehensive smart grid information on all aspects of perception. The grid system analysis, early warning, disaster preparedness and self-healing capabilities is improved. Smart Grid is an important application field of Things.

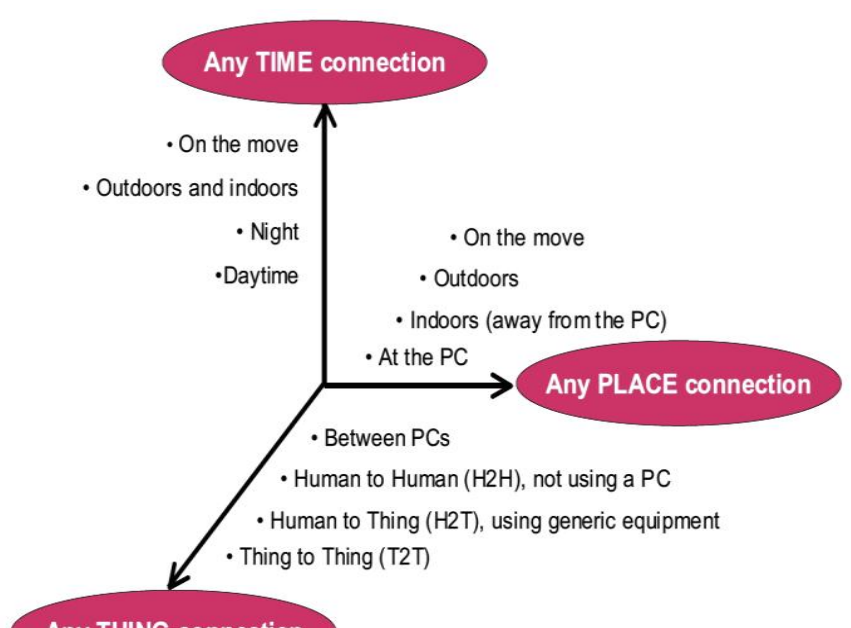

Any THING connection

Figure 1. Information exchange dimension

\subsection{Layered network structure of Internet of Things}

The Internet of Things application structure for smart grid cannot obtain timely. Demand service is crude. Within the system there are many isolated information islands lack of information sharing. Application in Internet of Things for smart grid is showed. Through the effective handling of the vast amounts of information, we can achieve the realtime monitoring and troubleshooting of transmission lines, substation equipment, distribution lines and distribution transformers, and deploy unified electric power resources to achieve with the user's 
information, thus achieve efficient, economic, safe, reliable and interactive smart grid.

Internet of Things applications functional framework for the smart grid proposed different application requirements according to different characteristics of the major aspects. According to the differences of performed functions and supporting technologies of different stages, combined with the basic network model of Internet of Things, Internet of Things for smart grid is divided into the perceived extension layer, network layer and application layer, three-layer network architecture, shown in Figure 2.

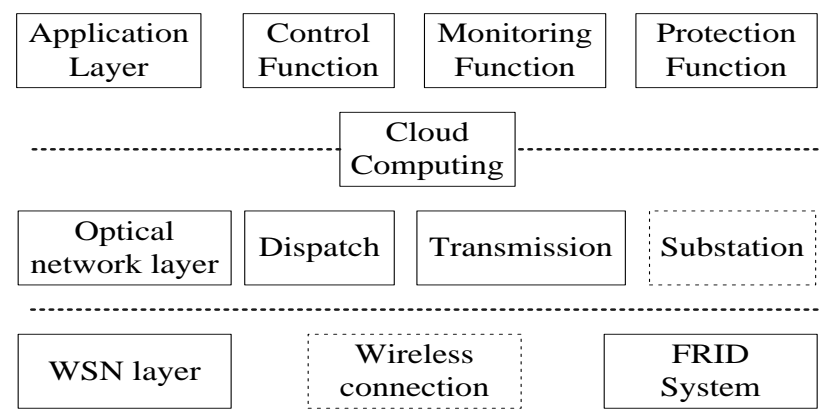

Figure 2. Information configuration of Internet of Things in smart grid

\subsection{Perception layer}

Perception layer mainly through radio transmitter, radio frequency identification technology and other means of power system for each application aspects related to information collection. The information collected from the perception object is classified and pretreated by wireless self-organizing sensor network, infrared communication, field-bus and other short-range communication equipments, in order to access to aware terminals and interactive terminals.

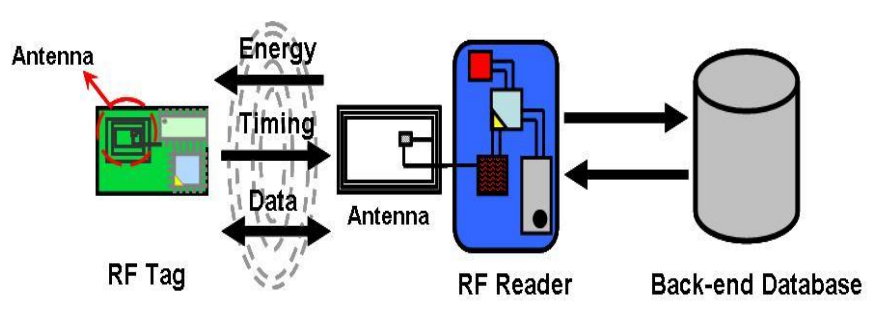

Figure 3. RFID composition

\subsection{Network layer}

The perceptive terminals and interactive terminals shield the differences between the various networks through the gateway and access to private network and the Internet according to data categories and level of security. Electricity access private network mainly includes power fiber-optic access networks and broadband wireless access networks which access to the power core network through the electric power private network, in order to achieve real-time data collection, reliable return. The side of Internet contains access methods.

\subsection{The application layer}

The application layer builds a variety of power application platforms for the business needs of smart grid. Companies are mutual perception and interaction with users through this platform. The connection of the perception interactive platform and the power core network must be isolated from each other, strong security measures to protect the indirect interconnection. Therefore, the two in the figure are connected with dotted line, which means a virtual interconnection under the conditions of physical isolation.

\section{KEY TECHNOLOGIES}

\subsection{Sensor technology}

Sensing technology is about to get information from natural sources, and the processing and recognition of a multi-disciplinary modern science and engineering. It relates to a sensor, planning and design of information processing and recognition, development, manufacture, testing, application and evaluation of improvement activities.

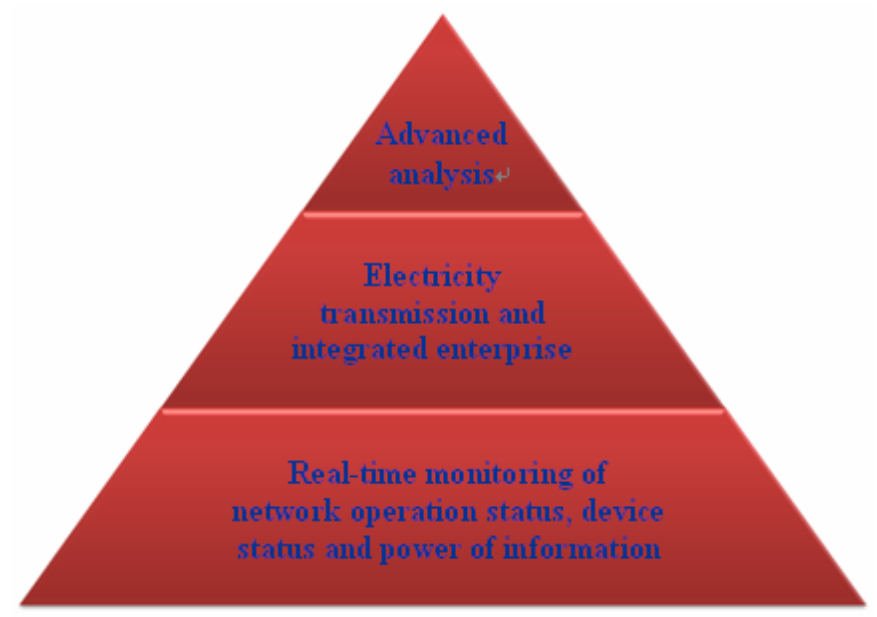

Figure 4. Three levels to improve grid reliability, efficiency and service level management

\subsection{Recognition technology}

Recognition technology mainly between items and code to establish one relationship through a certain coding rules and information carriers (such as electronic tags, bar code), and other items to achieve distinction and identification.

\subsection{Wireless sensor network technology}

Wireless sensor network technology is deployed in the detection area of a large number of low-cost micro-sensor nodes to form a self-organizing network technology system consisting of a multi-hop 
wireless communication, its purpose is cooperative sensing, collecting and processing the network coverage area is perceived information of the object, and send the viewer.

The wireless communication is a wireless communication technology using electromagnetic waves characteristic information number can be freely propagating in space and are a communication method information exchange. Wireless communication technology research focus include wireless broadband communication technology, short-range wireless communication technology, wireless base station wireless access technologies, wireless network optimization technology content group.

\subsection{Power communication technology}

Power communication technology is the use of power line transmission of a data and voice communication signals, then separated from the high-frequency current through the high-frequency signal containing information on the current load, and then use the power line transmission, the modem accepts the information, and transferred to a computer or a telephone, in order to achieve the transmission of information.

\subsection{Data processing technology}

Data processing technology with increasing the size of things, continuity and heterogeneous data volumes are huge challenges to deal with a large number of heterogeneous, mixed and incomplete data networking, cloud computing technologies need to be addressed, data mining, real-time database, massive computing.

\subsection{Visualization technology}

Visualization technology is the use of computer graphics and image processing technology, the data converted into graphics or images displayed on the screen and interact with the processing of the theory, methods and techniques.

\subsection{Spatial information technologies}

Spatial information technologies include satellite positioning systems, geographic information systems and remote sensing theory and technology, combined with computer technology and communication technology, spatial data acquisition, measurement, analysis, storage, management, display, dissemination and application.

\section{DEVELOPMENT OF THINGS}

\subsection{Development of Things in China}

At present, China has become the world's manufacturing center, the rapid development of information technology, material research networking technology is imminent. Things can be divided into three-tier architecture consisting perception layer, network layer and application layers.
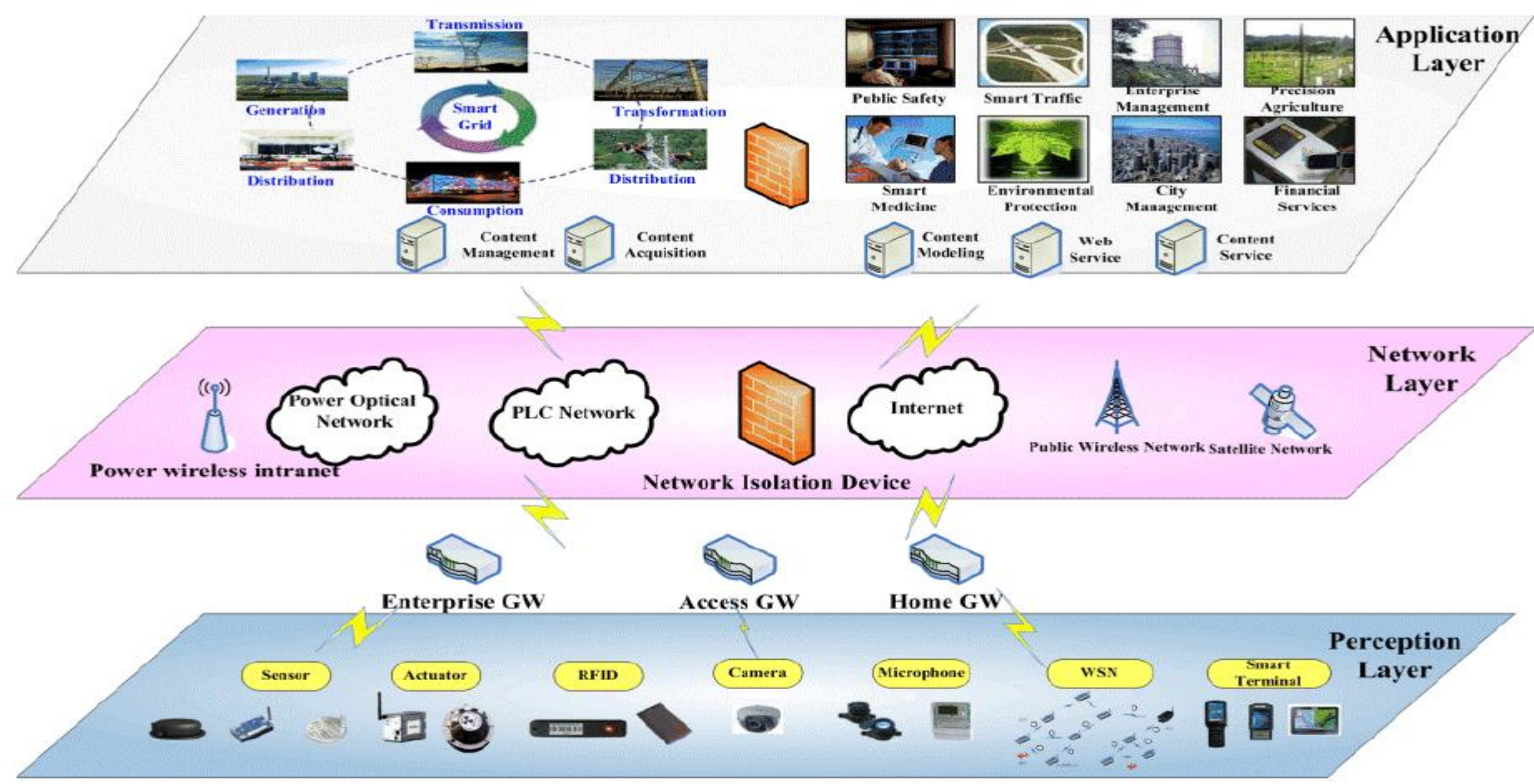

Figure 5. Things three reference architecture 
Overall perception, reliable transmission, intelligent processing capability is the core of things. Overall perception is the use of RFID, twodimensional code, GPS, cameras, sensors, sensor networks perception, capture, technical means to measure objects anywhere on the information collection and access. Reliable delivery is through a variety of communication networks and Internet convergence, the object access information network for reliable information exchange and sharing anytime, anywhere. Intelligent processing refers to the use of cloud computing, fuzzy recognition and other intelligent computing technology, the massive cross-regional, cross-industry, cross-sectoral analysis and processing of data and information to enhance the physical world, a variety of activities and changes in the economic and social insight force, intelligent decision-making and control.

\subsection{Development of Smart Grid in China}

Smart grid research started relatively late, from large power and low-voltage power grid cut two angles at the same time, relatively little has been fully mature and industrialization of the results. But research in a lot of area has laid a foundation for the development of smart grid.

The status quo of China's power grid construction, there are two obvious contradictions:

a. With the rapid development of China economy, the power consumption is growing very rapidly, arduous task to meet electricity demand.

b. Extremely uneven distribution of energy resources and economic development, as well as the existing power grid structure is not strong enough, greatly constrained economic development impact.

Consider the current situation of China's power grid construction, power grid construction with Chinese characteristics in production, the experience of European countries should formulate feasible routes and building a strong smart grid program.

\subsection{Things applications in the smart grid}

Researcher later information services in more developed countries, while in the tracking study has been developed gradually have their own innovations. Involved in research in this area has China ANCC, China Standards Association, AIM China, Fudan Auto. ID Labs China, Beijing University of Aeronautics and Astronautics and other research institutions and a number of enterprises, and achieved some initial results. At the same time, there are well-known domestic enterprises engaged in research and development of RFID in two sessions during 2005 submitted a "needs to adapt to social and economic development, the establishment of China Logistics Internet Project" proposal, put forward a proposal to carry out research and planning things Chinese, the current leaders of relevant ministries have been In this proposal, the inspection and verification.

Smart Grid application layer is the demand of networking technology combined with the smart grid, smart grid applications to achieve solutions. Smart Grid through the application layer and ultimately the depth of integration of information technology and the smart grid, has a broad impact on the development of smart grid. The key lies in the application layer in the information process, to meet all aspects of the grid system, intelligent communication, precise power, solar power, improve energy efficiency, security of supply, saving electricity costs each information element of target needs analysis and internal sharing of information.

\section{CONCLUSION}

The Internet of Things is one of the most important technological in terms of benefit. There are several critical elements to be improved in terms of both technologies and regulatory. Much of field trials are in progress around the world as well as researchers. Internet of Things is considered as the third revolution in the digital technology after the computer and the Internet. Currently, the power grid is transforming towards smart grid, the requirements of automation and intellectualization would lead to deep integrations of Internet of Things. The mature wireless communication theory and network optimization theory have been paved the way for theoretical basis. Among these there is the optimization of public light illumination. Generally speaking it is possible infer average values of economic benefits measured on the meter of 1 million habitants and organized by vertical sectors.

\section{REFERENCES}

[1] Jianming Liu, Xiangzhen Li, Xi Chen, Yan Zhen, Lingkang Zeng, Applications of Internet of Things on Smart Grid in China, International Conference on Advanced Communication Technology, 2011, 8(3): 13-17.

[2] Lee Yuanyuan; Miao Wei; Su Donglin; Tang Bihua. EMC test and frequency allocation of WSN used in spacecraft. 8th International Symposium on Antennas, Propagation and EM Theory.2008, 6(12): 1131-1134.

[3] Budka, K.; Deshpande, J.; Hobby, J.; Young-Jin Kim; Kolesnikov, V.; Wonsuck Lee; Reddington, T. GERI - Bell Labs Smart Grid Research Focus: Economic Modeling, Networking, and Security \& Privacy. 2010 First IEEE International Conference on Smart Grid Communications. 2010, 5(17): 208-213.

[4] Lee Yuanyuan; Miao Wei; Su Donglin; Tang Bihua. EMC test and frequency allocation of WSN used in spacecraft. 8th International Symposium on Antennas, Propagation and EM Theory. 2008, 4(116):1131-1134. 